\title{
Rate-Equation Approach to Laser Light Statistics ${ }^{1}$
}

\author{
L. Chusseau*, J. Arnaud**, and F. Philippe ${ }^{* * * * * * * *}$ \\ *Centre d'Électronique et de Micro-optoélectronique de Montpellier, UMR 5507 CNRS, \\ Université Montpellier II, F-34095 Montpellier, France \\ **Mas Liron, F-30440 Saint Martial, France \\ ***Départament Mathématiques et Informatique Appliqués, Université Paul Valéry, F-34199 Montpellier, France \\ **** Laboratoire d'Informatique, de Robotique et de Microélectronique de Montpellier, \\ UMR 5506 CNRS, F-34392 Montpellier, France \\ e-mail: chusseau@univ-montp2.fr; arnaudj2@wanadoo.fr; Fabrice.Philippe@univ-montp3.fr
}

Received November 25, 2002

\begin{abstract}
Single-mode cavity laser light statistics is considered within the framework of rate equations. According to this approach, fluctuations are caused by jumps in active and detecting atoms. The algebra is simple, allowing analytical expressions for the intracavity Fano factor and the photocurrent spectral density to be obtained. Poissonian, quiet, and optical pumps are considered. The results are verified by comparison with Monte Carlo simulations. An essentially exhaustive investigation of sub-Poissonian light generation by classical laser schemes, two-mode lasers, and semiconductor lasers is proposed. ( 2003 MAIK "Nauka/Interperiodica”.
\end{abstract}

\section{INTRODUCTION}

Light is called sub-Poissonian when the variance of the number of photodetection events counted over a large time duration is less than the average number of events. Equivalently, we may say that the photocurrent spectral density is below the shot-noise level at low Fourier (or baseband) frequencies. It has been shown experimentally by Machida et al. [1] that laser diodes driven by high-resistance electrical sources may generate sub-Poissonian light. This feature of great fundamental and practical importance, treated theoretically by Golubev and Sokolov [2] and Yamamoto et al. [3] on the basis of the laws of quantum optics may be understood alternatively as resulting from a birth-death Markov process efficiently modeled using Langevin forces and rate equations (see Arnaud $[4,5]$ for an introduction to the method).

The purpose of this paper is to apply the rate-equation approach to various laser schemes, namely, optically pumped three- and four-level lasers, two-mode lasers, and electrically pumped semiconductor lasers. The light statistics is calculated either analytically or using Monte Carlo techniques. The analytical expressions obtained from rate equations are found to coincide with those derived by quantum optics methods, but the algebra is considerably simpler. This is so even when the emitted light exhibits sub-Poissonian statistics. To wit, the expression for the internal cavity statistics of many four-level atoms with a negligible spontaneous decay previously given by Ritsch et al. [6] is recovered [7]. Similarly, expressions for three-level

\footnotetext{
${ }^{1}$ This article was submitted by the authors in English.
}

atoms obtained by Khazanov et al. [8] are recovered (see below). Coherently pumped three-level atom lasers [7] and two-mode lasers were apparently not treated earlier.

Rate equations treat the number of photons in the cavity as a classical random function of time. The light field is quantized as a result of matter quantization and conservation of energy, but not directly. Rate equations should be distinguished from semiclassical theories in which the optical field is driven by atomic dipole expectation values. The theory employed in this paper rests instead on the consideration of transition probabilities, as, for example, in the Loudon [9] treatment of optical amplifier noise. Every absorption event reacts To the number of light quanta in the optical cavity. Semiclassical theories are unable to explain sub-Poissonian light statistics because the light generation process and the light detection process are considered separately. Here, we treat the laser as a birth-death Markov process [10]. A Monte Carlo simulation gives the evolution of the number $m$ of photons in the cavity from which the Fano factor $\mathscr{F}=\operatorname{var}(m) /\langle m\rangle$ is obtained [7]. At the same time, the instants $t_{k}$ when photons are being absorbed provide the spectral density of the photocurrent whose normalized value $\mathscr{I}$ is unity for Poisson processes [11]. The normalized spectrum is denoted $\mathscr{I}(\Omega)$. The Fourier angular frequency $\Omega$ is called, for brevity, the frequency. When both the number of atoms and the pumping level increase, the computing time becomes prohibitively large because of the exponentially growing number of events to process. For simple laser schemes, analytical expressions are obtained by applying the weak-noise approximation. 
In Section 2, the rate-equation method is applied to V-type three-level lasers using both Monte Carlo simulations and the weak-noise approximation. The numerical method and the analytical method are found to agree for the range of parameters considered. More extensive treatments considering other laser schemes will appear elsewhere [7, 12]. Section 3 describes the complete treatment of a two-mode laser derived from a classical four-level scheme. Well-known experimental results relative to partition noise will be shown to follow from the coupled rate equations. Semiconductor lasers are treated in Section 4. The Monte Carlo simulation exhibits the combined influences of carrier heating and spectral hole burning on the dynamics of laser noise.

\section{A V-TYPE THREE-LEVEL LASER}

The active medium of V-type three-level lasers is a collection of $N$ identical atoms as depicted in Fig. 1. The level separations are supposed to be large compared with $k_{\mathrm{B}} T$, where $T$ denotes the optical cavity temperature and $k_{\mathrm{B}}$ the Boltzmann constant, so that thermally induced transitions are negligible.

The probability per unit time that an electronic transition from level $|1\rangle$ to level $|2\rangle$ occurs is taken as equal to $m$, and the probability of an electronic transition from $|2\rangle$ to $|1\rangle$ as $m+1$, where $m$ denotes the number of photons in the cavity. This amounts to selecting a time unit whose typical value depends on the medium gain. Spontaneous decay from level $|2\rangle$ to level $|1\rangle$ is allowed with probability $\gamma$. This decay may be either nonradiative or involve radiation into other electromagnetic modes, except for the one of interest.

Photons are absorbed with probability $\alpha m$, where $\alpha$ denotes a constant, the absorbing atoms residing most of the time in their ground state. These absorbing atoms model the transmission of light through mirrors with subsequent absorption by a detector. The detection provided is linear and reflectionless; it is immaterial whether absorption occurs inside or outside the optical cavity. As in the classical textbook by Sargent et al. [13], it is convenient to consider absorbing atoms located inside the cavity. For simplicity, internal absorption is neglected.

"Incoherent" pumping promotes electrons from level $|1\rangle$ to level $|3\rangle$ with probability $P$. The case of "coherent" pumping, which allows a fully symmetric electron exchange between levels $|1\rangle$ and $|3\rangle$, is discussed elsewhere [7]. When the pumping field originates from frequency-filtered thermal radiation, the pump fluctuations are nearly Poissonian.

Spontaneous decay from level $|3\rangle$ to the upper working level $|2\rangle$ occurs with probability $p_{u}$. All previously discussed relevant probabilities are schematized in Fig. 1.

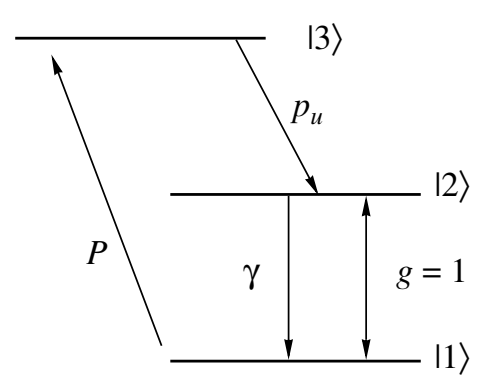

Fig. 1. V-type three-level laser under incoherent (unidirectional) optical pumping.

\subsection{Monte Carlo Model}

The rate-equation model of an $\mathrm{N}$-atom single-mode laser straightforwardly leads to a master equation for the probability of having $m$ photons stored in the cavity at time $t[5,9,14]$. Alternatively, the laser evolution is modeled as a temporally homogeneous birth-death Markov process. In the steady-state regime, rate of change and equilibrium probabilities of having $m$ and $m+1$ photons within the cavity are linked via a detailed balancing condition. This is a favorable condition for a Monte Carlo simulation [15], because every laser microstate belongs to a Markov chain and thus occurs proportionally to its equilibrium probability when the number of steps increases to infinity.

For V-type three-level lasers, rates of change $W_{j}$ are ascribed to the different kinds of events as given in the table.

For example, the probability that an atom jumps from level $|1\rangle$ to level $|3\rangle$ during the elementary time interval $t, t+\delta t$ is $W_{2} \delta t$, where $\delta t$ is chosen small enough so that this probability is much less than unity. Because atoms are coupled to one another only through the field, $W_{2}$ is proportional to the number $n_{1}$ of atoms in $|1\rangle$ at time $t$, and thus $W_{2}=P n_{1}$, where the constant $P$ is proportional to the pump strength. If a jump does occur, $n_{1}$ is reduced by 1 while the number $n_{3}$ of atoms in level $|3\rangle$ is incremented by 1 . If the initial value of $n_{3}$ is $N$, the event does not occur. Similar considerations apply to the other jump probabilities. Note that the coherent emission rate $W_{3}$ is proportional to $m+1$, fol-

Elementary events in V-type 3-level lasers (see Fig. 1) and corresponding rate of change $W_{j}$

\begin{tabular}{l|c|l}
\hline \multicolumn{1}{c|}{ Event } & Transition & \multicolumn{1}{c}{ Rate } \\
\hline Photon absorption & - & $W_{1}=\alpha m$ \\
Pump absorption & $|1\rangle \longrightarrow|3\rangle$ & $W_{2}=P n_{1}$ \\
Coherent emission & $|2\rangle \longrightarrow|1\rangle$ & $W_{3}=(m+1) n_{2}$ \\
Coherent absorption & $|1\rangle \longrightarrow|2\rangle$ & $W_{4}=m n_{1}$ \\
Spontaneous decay & $|2\rangle \longrightarrow|1\rangle$ & $W_{5}=\gamma n_{2}$ \\
Upper decay & $|3\rangle \longrightarrow|2\rangle$ & $W_{6}=n_{3} p_{u}$ \\
\hline
\end{tabular}




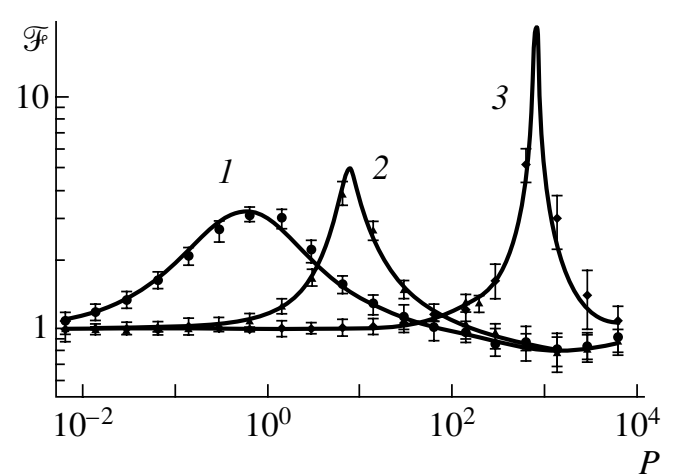

Fig. 2. Intracavity Fano factor for V-type lasers as a function of the pumping rate $P$. Error bars are the $95 \%$ confidence level from a statistical treatment applied to ten Monte Carlo runs, each having duration $T_{m}=200$. Solid lines are analytical. The parameters are $N=100 ; p_{u}=632 ; \alpha=6.32$; and $\gamma=(1) 0$, (2) 6.32, and (3) 632 .

lowing the Einstein prescription. This ensures that laser emission restarts if extinction occurs. A key feature that distinguishes the present formulation from other rateequation methods is that absorption of photons by the detector is included in the system description. Because detection is supposed to be linear, such events are taken to occur with a rate $W_{1}=\alpha m$, where $\alpha$ expresses detector absorption.

An efficient algorithm has actually been employed [10]. Given that an event of any kind occurred at time $\tau_{k}$, the time of the next event is

$$
\tau_{k+1}=\tau_{k}+\frac{1}{\sum_{j} W_{j}} \ln \left(\frac{1}{r}\right),
$$

where $\mathfrak{r}$ is a random number uniformly distributed in the interval $[0,1]$. The probability that the event is of kind $l$ is equal to $W_{l} / \sum_{j} W_{j}$. The Monte Carlo method is readily implemented; only the total number of atoms in each state needs to be tracked (for fermions, the numerical procedure is significantly more involved [11]). Within the whole time set $\left\{\tau_{k}\right\}$, we select the subset $\left\{t_{k}\right\}$ of photonabsorption events. It is then straightforward to evaluate the photo-detection noise spectrum.

The intracavity Fano factor $\mathscr{F}$ is represented in Fig. 2. The analytical results of Section 2.2 agree well with the simulation. Note that $\mathscr{F}$ is below unity within some pumping range. This behavior is in good agreement with previous quantum-optics results $[6,8,16$, 17].

The normalized spectral density $\mathscr{I}(\Omega)$ is represented in Fig. 3 for two sets of parameter values. For each Monte Carlo run, $\mathscr{I}(\Omega)$ is first evaluated from the $\left\{t_{k}\right\}$ list [11] and refined using a smoother power spectral density estimator [18, 19]. Averaging over runs and concatenating neighboring frequencies produce the

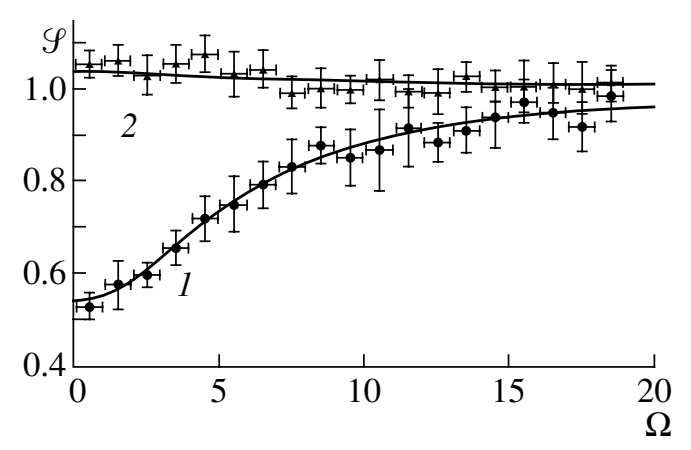

Fig. 3. Normalized photocurrent spectral density $\mathscr{I}$ of Vtype lasers as a function of Fourier frequency $\Omega$. Points with error bars are from Monte Carlo simulations with 150 runs, each of duration $T_{m}=100$. Solid lines are analytical. The parameters are $N=100 ; p_{u}=632 ; \alpha=6.32 ;$ and $(1) \gamma=0$ and $P=1265 ;(2) \gamma=6.32$ and $P=20$.

final data, together with error bars at the $95 \%$ confidence level. There is fair agreement between Monte Carlo simulations and analytical formulas to be subsequently reported. Both predict sub-Poissonian photocurrent statistics. Even with one billion photon-absorption events, Monte Carlo spectra exhibit large error bars. An analytical method is to be preferred when it exists. At the same time, Monte Carlo simulations do not rely on linearization and provide a useful check.

\subsection{Analytic Model}

Let us first set up the steady-state conditions. If $n_{j}$, $j=1,2,3$, denote the number of atoms in state $j$, we have

$$
n_{1}+n_{2}+n_{3}=N \text {. }
$$

Let $\mathscr{F}$ denote the net pumping rate, $\mathscr{R}$ the net stimulated rate, $\mathcal{U}$ the upper decay rate, $\mathscr{Y}$ the spontaneous decay rate from the upper to the lower working levels, and 2 the photon absorption rate. The steady-state conditions then read

$$
\begin{gathered}
\mathscr{S}=\mathscr{U}=\mathscr{D}=\mathscr{R}+\mathscr{S}, \\
2=\mathscr{R},
\end{gathered}
$$

where

$$
\begin{gathered}
\mathscr{S}=P n_{1}, \quad \mathscr{U}=p_{u} n_{3}, \\
\mathscr{S}=\gamma n_{2}, \quad \mathscr{Q}=\alpha m, \\
\mathscr{R}=(m+1) n_{2}-m n_{1} .
\end{gathered}
$$

A straightforward solution of Eqs. (2)-(4) provides the steady-state atomic populations $n_{i}$ and photon number $m$.

Within the weak-noise approximation, populations and rates are split into steady-state values and fluctuations. The instantaneous photon number $m$ is thus writ- 
ten as $\langle m\rangle+\Delta m$, where $\langle m\rangle$ denotes the steady-state value. For rates, fluctuations consist of a deterministic function and uncorrelated Langevin "forces"; i.e., $J=$ $\langle\mathscr{F}\rangle+\Delta J$, the $\Delta J$ term including a Langevin force $j(t)$ expressing the randomness of the jump process; thus,

$$
\begin{gathered}
\mathscr{J} \equiv J+\Delta J, \quad \mathscr{Q} \equiv U+\Delta U, \\
\mathscr{S} \equiv S+\Delta S, \quad 2 \equiv Q+\Delta Q, \\
\mathscr{R} \equiv R+\Delta R,
\end{gathered}
$$

where a first-order variation of the expressions in Eq. (4) yields

$$
\begin{aligned}
\Delta J & =P \Delta n_{1}+j, \\
\Delta R=(m+1) \Delta n_{2} & -m \Delta n_{1}+\left(n_{2}-n_{1}\right) \Delta m+r, \\
\Delta S & =\gamma \Delta n_{2}+s, \\
\Delta U & =p_{u} \Delta n_{3}+u, \\
\Delta Q & =\alpha \Delta m+q .
\end{aligned}
$$

Similarly, a first-order variation of the population conservation rule gives

$$
0=\Delta n_{1}+\Delta n_{2}+\Delta n_{3} .
$$

First, let us consider zero-frequency noise. Eq. (3), when applied to variations, reads

$$
\begin{gathered}
\Delta J=\Delta U=\Delta R+\Delta S, \\
\Delta Q=\Delta R .
\end{gathered}
$$

By replacing atomic populations and photon numbers by their steady-state values, the above set of equations is solved. In particular, $\Delta Q$ is obtained as a linear combination of the Langevin forces,

$$
\Delta Q=\sum_{z \in\{j, u, q, r, s\}} c_{z} z,
$$

where the $c_{z}$ are real coefficients that depend on the parameters $N, P, p_{u}, \gamma$, and $\alpha$. The detailed expressions, too lengthy to be given here in their general form, are conveniently handled using symbolic calculations.

The normalized zero-frequency photocurrent spectral density is of the form

$$
\Phi=\frac{1}{\alpha m} \sum_{z \in\{j, u, q, r, s\}} c_{z}^{2} \sigma_{z},
$$

where $\sigma_{z}$ denotes the spectral density value of the Langevin noise source $z$, equal to average rates,

$$
\begin{gathered}
\sigma_{j}=P n_{1}, \quad \sigma_{u}=p_{u} n_{3}, \\
\sigma_{s}=\gamma n_{2}, \quad \sigma_{q}=\alpha m, \\
\sigma_{r}=(m+1) n_{2}+m n_{1} .
\end{gathered}
$$

When these expressions are introduced in Eq. (10), an analytical expression of $\mathscr{I}$ is obtained.

Consider now the very special case where $\gamma=0$ and $N \gg \alpha$, i.e., the laser is thresholdless and has very low loss. The normalized photocurrent spectral density reads

$$
\Phi=1-\frac{4 P p_{u}}{\left(P+2 p_{u}\right)^{2}} .
$$

$\mathscr{I}$ is thus unity at low and high pumping levels and goes to a minimum in between these bounds. The minimum value, $\mathscr{I}_{\text {min }}$, and the corresponding pumping value are

$$
\Phi_{\text {min }}=\frac{1}{2}, \quad P=2 p_{u} .
$$

It can be shown that this minimum is the absolute minimum of $\mathscr{I}$, irrespectively of the values of $\gamma, N$, and $\alpha$ [7].

A similar technique applied to $\Lambda$-type three-level lasers and four-level lasers leads to $\mathscr{\Phi}_{\text {min }}=1 / 2$ and $\Phi_{\text {min }}=1 / 3$, respectively. These values are in complete agreement with three-level theories proposed earlier by Khazanov et al. [8] and by Ralph and Savage [16, 20] and with the four-level theory of Ritsch et al. [6].

At some Fourier frequency $\Omega$, the generalized rate equations read [4]

$$
\begin{gathered}
i \Omega \Delta m=\Delta R-\Delta Q, \\
i \Omega \Delta n_{1}=\Delta R+\Delta S-\Delta J, \\
i \Omega \Delta n_{2}=\Delta U-\Delta R-\Delta S, \\
i \Omega \Delta n_{3}=\Delta J-\Delta U .
\end{gathered}
$$

Again, we solve for $\Delta Q$ the linear system of fluctuations and obtain for the normalized spectral density

$$
\mathscr{S}(\Omega)=\frac{1}{\alpha m} \sum_{z \in\{j, u, q, r, s\}} \tilde{c}_{z}(\Omega) c_{z}^{*}(\Omega) \sigma_{z},
$$

where the coefficients $\tilde{c}_{z}$ are complex and frequencydependent and where the Langevin "forces" $\sigma_{z}$ are still given in Eq. (11).

After rearranging, Eq. (15) gives the spectral density in the form

$$
\mathscr{I}(\Omega)=1+\frac{a_{2} \Omega^{4}+a_{1} \Omega^{2}+a_{0}}{\Omega^{6}+b_{2} \Omega^{4}+b_{1} \Omega^{2}+b_{0}},
$$

where the coefficients $a_{i}$ and $b_{i}$ are real. The form in Eq. (16) ensures that $\Phi(\Omega)$ tends to shot-noise level at high frequencies. Figure 4 shows that $\mathscr{I}(\Omega)$ reaches its minimum value at $\Omega=0$.

When spontaneous decay from the upper working level may be neglected (Fig. 4a), light is always subPoissonian and the lowest $\mathscr{I}$ value occurs when $P / p_{u}=$ 2 . Figure $4 \mathrm{~b}$ illustrates the fact that spontaneous decay 

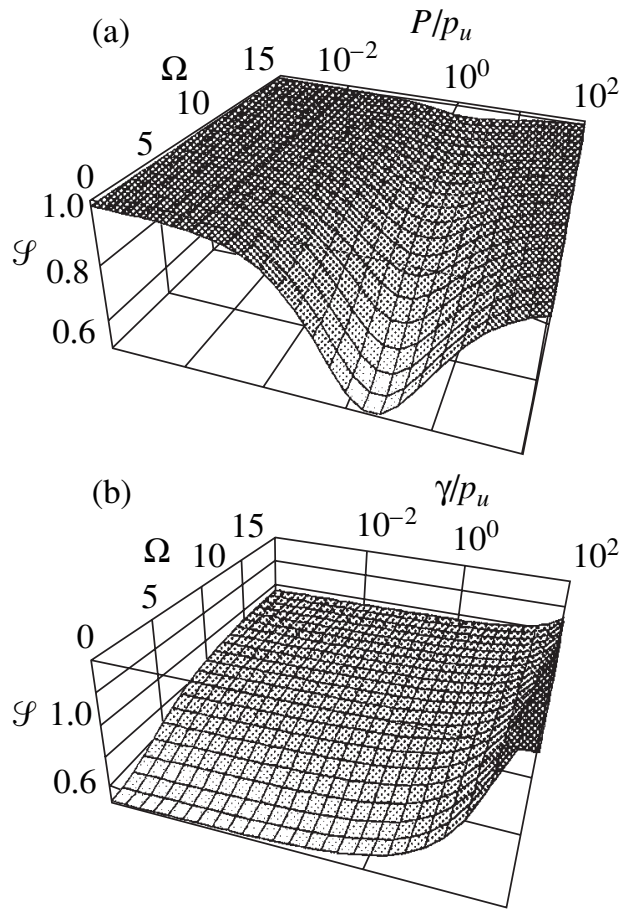

Fig. 4. 3D plot of $\mathscr{I}$ of a V-type laser as a function of Fourier frequency $\Omega$ and (a) normalized pumping rate $P / p_{u}$ and (b) normalized recombination rate $\gamma / p_{u}$. The laser parameters are $N=10^{5}, \alpha=6.32$, and $p_{u}=632$. The more sub-Poissonian the light statistics, the lighter the surface.

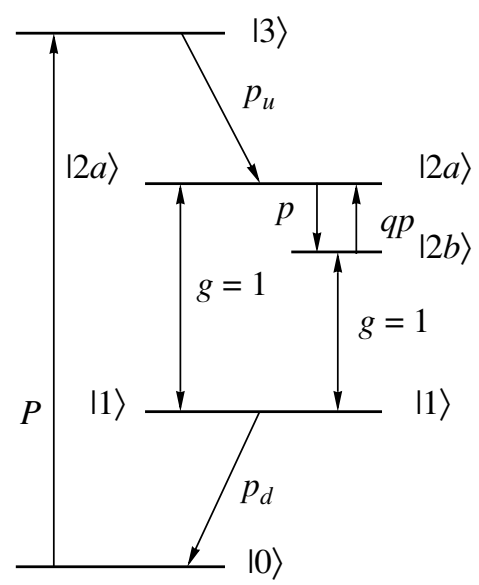

Fig. 5. Two-mode laser scheme.

from the upper working level is inconsequential until $\gamma / p_{u} \approx 3 \times 10^{-2}$. However, the light statistics ceases to be sub-Poissonian when $\gamma / p_{u}>0.3$ because the lasing threshold can no longer be reached.

The intracavity photon statistics is characterized by the Fano factor $\mathscr{F}=\left\langle\Delta m^{2}\right\rangle /\langle m\rangle$, where $m$ denotes as before the number of photons in the cavity. The variance of $m$ equals the integration over frequency of $\Phi_{\Delta m}(\Omega)$, the normalized spectral density of $\Delta m$. As previously, $\mathscr{T}_{\Delta m}(\Omega)$ is obtained by solving for $\Delta m$ instead of $\Delta Q$ and its general form is

$$
\mathscr{I}_{\Delta m}(\Omega)=\frac{a_{2}^{\prime} \Omega^{4}+a_{1}^{\prime} \Omega^{2}+a_{0}^{\prime}}{\Omega^{6}+b_{2}^{\prime} \Omega^{4}+b_{1}^{\prime} \Omega^{2}+b_{0}^{\prime}},
$$

where $a_{i}^{\prime}$ and $b_{i}^{\prime}$ are real coefficients. The Fano factor is thus

$$
\mathscr{F}=\int_{-\infty}^{\infty} \mathscr{I}_{\Delta m}(\Omega) \frac{d \Omega}{2 \pi} .
$$

It has already been illustrated in Fig. 2 and successfully compared to Monte Carlo calculations.

\section{A TWO-MODE LASER}

Consider now a two-mode laser and let us focus on normalized spectral densities of each individual mode and of the total laser emission. As shown in Fig. 5, the scheme starts from the four-level laser. The upper lasing level $|2\rangle$ has been split into two sublevels $|2 a\rangle$ and $|2 b\rangle$, both being able to relax radiatively toward level $|1\rangle$. The optical gains of these two transitions are kept identical.

The beating between the two optical modes is arbitrarily prescribed at a frequency of $1 \mathrm{THz}$. The corresponding energy splitting is thus $\varepsilon=4.1 \mathrm{meV}$. The coupling $|2 a\rangle \longrightarrow|2 b\rangle$ is ensured by a transfer parameter $p$. The coupling $|2 b\rangle \longrightarrow|2 a\rangle$ is slightly less, e.g., $q p$. As driven by temperature it refills level $|2 a\rangle$ from $|2 b\rangle$ according to Boltzmann law, $q=\exp \left(-\varepsilon / k_{\mathrm{B}} T\right)$. Atomic populations are supposed to be unable to respond to the very high modal beat frequency.

It is straightforward to identify physical processes and corresponding rates by analogy with the discussion in Section 2. Let us, moreover, assume a weak coupling between the two modes just as in the textbook of Sargent et al. [13]. Thus, both modes truly exist. For the sake of simplicity, non-radiative relaxations $|2 a\rangle \longrightarrow$ $|1\rangle$ and $|2 b\rangle \longrightarrow|1\rangle$ are neglected and the photon count of each mode is supposed to be far greater than unity, $m_{a} \gg 1$ and $m_{b} \gg 1$. The rates thus read

$$
\begin{gathered}
\mathscr{\mathscr { T }}=P n_{0}, \quad \mathscr{U}=p_{u} n_{3}, \\
\mathscr{R}_{a}=m_{a}\left(n_{2 a}-n_{1}\right), \quad \mathscr{T}=p n_{2 a}-q p n_{2 b}, \\
\mathscr{R}_{b}=m_{b}\left(n_{2 b}-n_{1}\right), \quad \mathscr{D}=p_{d} n_{1}, \\
\mathscr{Q}_{a}=\alpha m_{a}, \quad \mathscr{Q}_{b}=\alpha m_{b} .
\end{gathered}
$$

The stable two-mode steady-state solution is calculated using conservation rules for populations and rates. Of 
major interest are the modal steady-state photon numbers

$$
\begin{gathered}
m_{b}=\frac{(1-q) p\left(P(N+\alpha) p_{u}+\alpha p_{d}\left(P+p_{u}\right)\right)}{\alpha\left(3 P p_{u}+p_{d}\left(P+p_{u}\right)\right)}, \\
m_{a}=\frac{P p_{d}\left(p_{u}(N-2 \alpha)-\alpha p(1-q)\right)}{\alpha\left(3 P p_{u}+p_{d}\left(P+p_{u}\right)\right)}-m_{b} .
\end{gathered}
$$

Figure 6 represents the average number of photons in the cavity for a particular choice of $p=4 p_{u}$ that roughly balances the two mode powers. As for optically pumped single-mode lasers [7], $m$ increases regularly with pump intensity and saturates at a very high level because of ground-state population depletion.

As was done before in Section 2.2, total rates and populations are split into average values and variations. We evaluate the normalized zero-frequency photocurrent spectral densities for each mode and for the total laser output. Figure 7 gives plots of photodetection spectral densities of each mode and of the total emitted light. Again, the parameter is the pumping $P$.

The total photodetection spectral density is nearly the same as for single-mode four-level lasers. Here again, sub-Poissonian behavior is observed at high pumping levels [7]. It can be shown that, when $\alpha$ tends to $0, \mathscr{S}$ reduces to

$$
\mathscr{I}=\frac{9 P^{2} p_{u}^{2}+p_{d}^{2}\left(P^{2}+p_{u}^{2}\right)}{\left(3 P p_{u}+p_{d}\left(P+p_{u}\right)\right)^{2}},
$$

which admits the minimum value $\mathscr{I}_{\min }=1 / 3$ if $P=p_{u}=$ $p_{d} / 3$. Although pumping conditions are slightly different, the $\Phi_{\min }$ value is the same as for usual four-level lasers $[6,7]$.

The most important feature of Fig. 7 appears on the individual mode spectral densities $\mathscr{\Phi}_{a}$ and $\mathscr{I}_{b}$, which are much more "noisy" than the previous total spectral density $\mathscr{I}$. Moreover, any attempt to balance mode powers maximizes $\mathscr{I}_{a}$ and $\mathscr{I}_{b}$. This effect, usually referred to as the mode-partition noise, has often been observed. It is very easily and straightforwardly explained by the present rate-equation approach.

\section{A SEMICONDUCTOR LASER}

Actual semiconductor lasers are complex systems. An idealized model will prove helpful. One-electron energy levels in the semiconductor are supposed to be of the form $\epsilon_{k}=k \epsilon$, with $k$ an integer and $\varepsilon$ a constant; e.g., $\varepsilon=1 \mathrm{meV}$. Allowed levels may be occupied by at most one electron, to comply with the Pauli exclusion principle. The electron spin, ignored in the present paper for the sake of brevity, is discussed, for example, in [21]. Our model considers neither electronic superposition states nor any strong Coulomb interaction, approximations made in virtually all laser-diode theories. In semiconductors, the allowed electronic levels

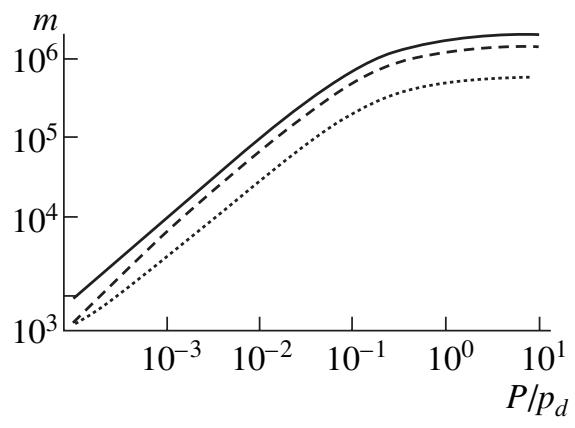

Fig. 6. Average number of photons in the cavity as a function of the normalized pumping level $P / p_{d}$. Dotted line: $m_{b}$, transition $|2 b\rangle \longrightarrow|1\rangle$; dashed line: $m_{a}$, transition $|2 a\rangle \longrightarrow|1\rangle$; solid line: $m=m_{a}+m_{b}$ (total of both transitions). Laser parameters: $N=10^{5}$ atoms, $p_{d}=632, p_{u}=316$, $\alpha=6.32, p=1265, T=300 \mathrm{~K}$.

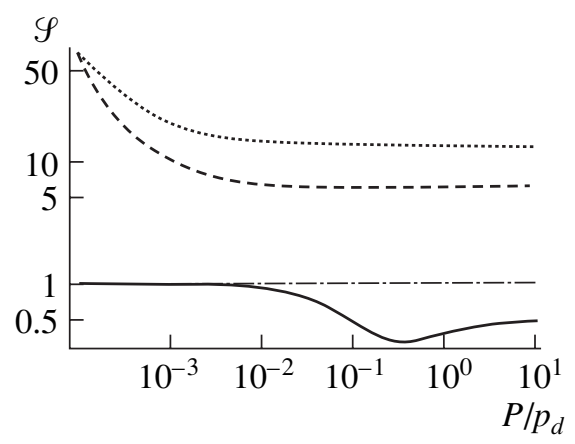

Fig. 7. Normalized spectral density, $\mathscr{I}$, of the two-mode laser. Line symbols and laser parameters are the same as in Fig. 6 except for the dotted-and-dashed line that represents the shot-noise level.

group into two bands, the conduction band (CB) and the valence band (VB). We suppose that both bands involve the same number of levels. The band-gap energy is instrumental in determining the laser oscillation frequency, but it will not enter into our model, because of simplifying assumptions to be discussed later. The number of electrons matches the number of allowed energy levels, and thus the electron-lattice system is electrically neutral. For pure semiconductors at $T=0 \mathrm{~K}$, the $N$ electrons fill up the valence band while the conduction band is empty.

The rate-equation approach first requires the identification of the elementary Markov processes that electrons and photons follow. Photon absorption is supposed to be only due to detecting atoms, that is, no additional optical loss is considered. Detecting atoms are assigned a probability $\alpha m$ of being promoted to the upper state, where $\alpha$ denotes a constant and $m$ the number of photons in the cavity. Stimulated absorption occurs with a probability $m$ for electrons in the lower 


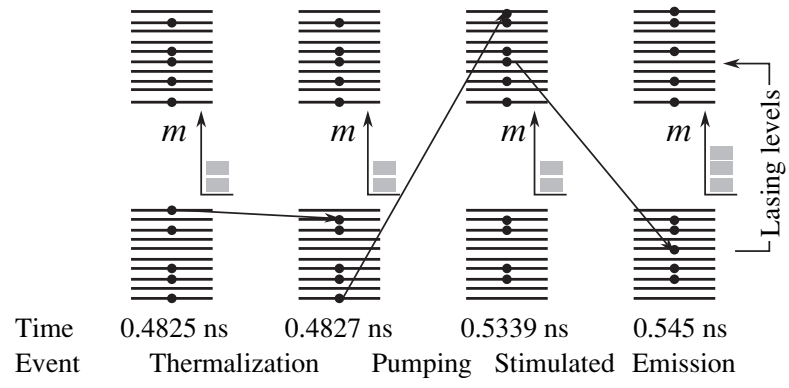

Fig. 8. Four-frame sequence illustrating the Monte Carlo simulation of a semiconductor laser. Ten equally spaced energy levels are considered in the VB and the CB. The insets represent the number of photons $m$ stored in the cavity at a given time by stacked blocks. Arrows show the movements of an electron from one energy level to another.

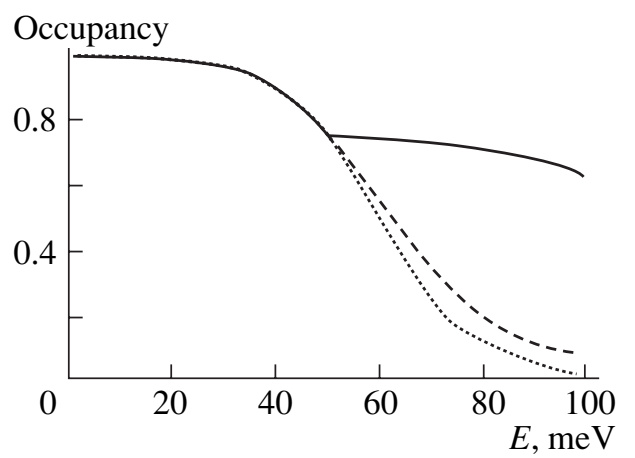

Fig. 9. Monte Carlo electron occupancy in the $\mathrm{CB}$ as a function of the energy position $E$ (reckoned from the bottom of the $\mathrm{CB}$ ) and the thermalization rate $p$. The lasing level is $E=50 \mathrm{meV}$. Dotted line: $p=25000 \mathrm{~ns}^{-1}$; dashed line: $p=$ $1000 \mathrm{~ns}^{-1}$; solid line: $p=250 \mathrm{~ns}^{-1}$.

working level to be promoted to the upper working level. Stimulated emission is modeled by assigning a probability $m+1$ to electrons in the upper working level to be demoted to the lower working level. Setting as unity the factor that multiplies the expressions $m$ or $m+1$ amounts to selecting a time scale, e.g., $1 \mathrm{~ns}$. In addition, the laser is assumed to be single-mode and lasing is thought to take place between levels located in the middle of the CB and the VB.

Only perfectly regular electrical pumping is considered. Since output photons reproduce (for slow variations) the electrical source statistics, the laser is expected to exhibit sub-Poissonian light statistics at low baseband frequencies [2]. Practical realizations involve electrical current generated by a cold highimpedance electrical source, which is almost nonfluctuating, as a consequence of the Nyquist theorem [3]. In the model, quiet electrical pumping is obtained by promoting low-lying electrons into high-lying levels periodically over time. As long as this time period remains short as compared to the time scales of interest, this prescription implies that the pumping rate is nearly constant.

Let us now consider the process of thermalization between the electron gas and the lattice. To enforce thermalization, each electron is ascribed a probability $p$ per unit time of being demoted to the adjacent lower level provided this level is empty, and a probability $q p$, where $q=\exp \left(-\varepsilon / k_{\mathrm{B}} T\right)$ of being promoted to the adjacent upper level if it is empty. If $p$ is large, thermalization is very efficient and electron-gas temperatures in both bands are equal to the lattice temperature. An analytic expression of the normalized spectral density can be obtained in this case $[11,22]$ :

$$
\Phi(\Omega)=1+\frac{\frac{\alpha(1+\alpha)}{1-\alpha} \frac{F}{J^{*}}-1}{\frac{1-\alpha^{2}}{2 \alpha^{2}} J^{*} F+(1-F)^{2}},
$$

where $F=\Omega^{2} / \Omega_{r}^{2}, \Omega_{r}^{2}=\left(1-\alpha^{2}\right) J^{*} / 2, J^{*}=(J \varepsilon) /\left(k_{\mathrm{B}} T\right)$, $J$ is the pumping rate in electrons per second, and $\alpha$ denotes the cavity losses. To the contrary, if $p$ is not large, electron-gas temperatures are ill-defined and the electronic populations are out of thermal equilibrium. Up to a point, a decrease $p$ is equivalent to an increase in the pumping rate.

The above description of semiconductor lasers permits Monte Carlo calculation as depicted with the four successive frames given in Fig. 8. Three elementary processes are illustrated from a computer simulation involving only ten levels in each band. At the start, the system has already reached a stationary regime. A sample of the electron distribution is shown on the left. The corresponding time and the number of light quanta stored in the cavity are, respectively, $\tau_{0}=0.4825 \mathrm{~ns}$ and $m=2$. The first event, at $\tau_{1}=0.4827 \mathrm{~ns}$, is a VB thermalization. Its effect is to decrement the system energy by $\varepsilon$, since an electron is demoted by one energy step. The second event, at $\tau_{2}=0.5339 \mathrm{~ns}$, is an electrical pumping event that promotes the VB electron occupying the lowest energy level to the highest energy level of the CB. The third event illustrates stimulated emission between the lasing levels at $\tau_{3}=0.545 \mathrm{~ns}$. As a result, the number of light quanta is incremented from $m=2$ to $m=3$.

The results of the Monte Carlo calculations are given in Figs. 9 and 10, where $p$, the rate at which thermalization events occur, is taken as the main parameter of interest. The laser model includes 100 levels evenly spaced by $\varepsilon=1 \mathrm{meV}$ in each band. A lattice temperature of $T=100 \mathrm{~K}$ is assumed, thereby yielding $q \approx 0.89$. The regular pumping transfers one VB electron to the $\mathrm{CB}$ at a rate of $5 \mathrm{~ns}^{-1}$.

The occupancy in the CB is plotted in Fig. 9 as a function of energy level $E$, referred to the bottom of the 


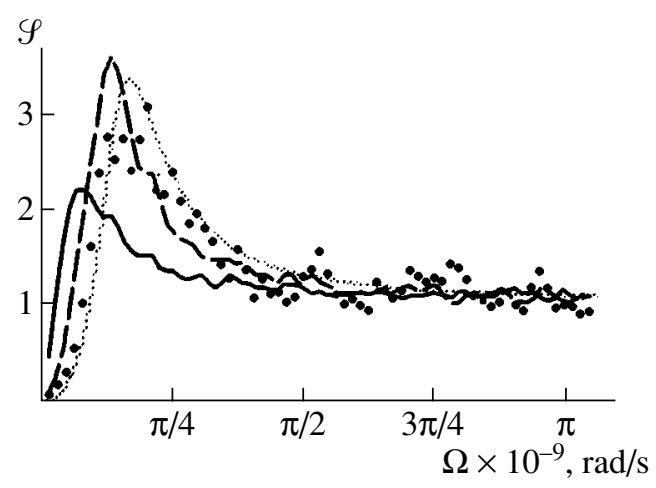

Fig. 10. Monte Carlo normalized spectral density $\mathscr{I}$ as a function of the normalized Fourier angular pulsation $\Omega$. The gray curve is the analytical calculation assuming perfect thermalization from Eq. (22). Dotted line: $p=25000 \mathrm{~ns}^{-1}$; dashed line: $p=1000 \mathrm{~ns}^{-1}$; solid line: $p=250 \mathrm{~ns}^{-1}$.

CB. As expected, a decrease in $p$ (or an increase in the pumping) modifies the CB occupancy. The FermiDirac (FD) statistic is recovered at a high $p$ value, a regime where the analytical model of Eq. (22) is in good agreement with the Monte Carlo calculations. It is worth noting that sub-Poissonian light statistics is achieved at low baseband frequencies, up to $\Omega \approx 0.1$.

At moderate $p$ values, or, equivalently, at moderate pumping levels, the occupancy slightly departs from FD law and the frequency domain of sub-Poissonian light emission is reduced. A FD fit of the occupancy (the dashed line in Fig. 9) gives a carrier temperature of $T=132 \mathrm{~K}$. Inserting this revised electron-gas temperature into Eq. (22) does not suffice to explain the Monte Carlo results. In our opinion, spectral hole burning (SHB) may be responsible for the obtained spectrum, even though this effect does not appear conspicuously on the occupancy curves. As a secondary effect, the range of $\Omega$ over which light is sub-Poissonian is reduced by approximately $40 \%$.

At very high pumping rates, the SHB is conspicuous on the solid line of Fig. 9 and the range of $\Omega$ where subPoissonian light is observed is reduced by approximately $80 \%$. The FD statistics evidently no longer apply and $\mathscr{I}(\Omega)$ is no longer described by Eq. (22). These Monte Carlo results show that SHB and carrier thermalization are physical phenomena that one cannot separate. Both act on the fluctuation spectra and the laser dynamics with the consequence of a reduction of the sub-Poissonian emission range.

\section{CONCLUSIONS}

The rate-equation approach was applied to various laser schemes. An accurate description of laser light statistics was obtained in every case, even when the laser light emission is sub-Poissonian because of a driv- ing quiet pump or because of the depletion of the lower level by an intense optical pump.

For simple laser schemes, fully analytical expressions were derived for the output light spectral density and the intracavity Fano factor. These expressions coincide with already published results that were available only for some laser schemes (three- and four-level lasers) and special values of the parameters (no spontaneous recombination rate, infinitely low optical losses). At the same time, the microscopic Markov approach underlying rate equations was exploited in Monte Carlo calculations. It was demonstrated to be fully equivalent to analytical calculations considering the V-type threelevel laser. A far more complicated laser scheme was considered in Monte Carlo calculations, namely a quiet-pump semiconductor laser. The method offers new insights concerning the combined influence of the carrier thermal heating and spectral hole burning that occur when pumping increases. Particularly, the reduction of the sub-Poissonian light emission range was demonstrated. Earlier work [23] has shown that the rate-equation method also enables one to treat phase fluctuations, excess noise, nonlinear gain, multiphoton processes, and electronic feedbacks from detectors to modulators.

\section{ACKNOWLEDGMENTS}

This work was supported by the STISS Department of Université Montpellier II and by CNRS under the JemSTIC Program.

\section{REFERENCES}

1. S. Machida, Y. Yamamoto, and Y. Itaya, Phys. Rev. Lett. 58, 1000 (1987).

2. Y. M. Golubev and I. V. Sokolov, Zh. Éksp. Teor. Fiz. 87, 408 (1984) [Sov. Phys. JETP 60, 234 (1984)].

3. Y. Yamamoto, S. Machida, and O. Nilsson, Phys. Rev. A 34, 4025 (1986).

4. J. Arnaud, Opt. Quantum Electron. 27, 63 (1995).

5. J. Arnaud, Opt. Quantum Electron. 34, 393 (2002); quant-ph/0201151.

6. H. Ritsch, P. Zoller, C. W. Gardiner, and D. F. Walls, Phys. Rev. A 44, 3361 (1991).

7. L. Chusseau, J. Arnaud, and F. Philippe, Phys. Rev. A 66, 053818 (2002).

8. A. M. Khazanov, G. A. Koganov, and E. O. Gordov, Phys. Rev. A 42, 3065 (1990).

9. R. Loudon, The Quantum Theory of Light (Oxford Univ. Press, Oxford, 1983).

10. D. T. Gillespie, Markov Processes: An Introduction for Physical Scientists (Academic, San Diego, 1992).

11. L. Chusseau and J. Arnaud, Opt. Quantum Electron. 34, 1007 (2002).

12. L. Chusseau, J. Arnaud, and F. Philippe, Opt. Commun. 213, 325 (2002).

13. M. Sargent III, M. O. Scully, and W. E. Lamb, Jr., Laser Physics (Addison-Wesley, Reading, MA, 1974). 
14. M. O. Scully and W. E. Lamb, Jr., Phys. Rev. 159, 208 (1967).

15. D. P. Landau and K. Binder, A Guide to Monte Carlo Simulations in Statistical Physics (Cambridge Univ. Press, Cambridge, 2000).

16. T. C. Ralph and C. M. Savage, Phys. Rev. A 44, 7809 (1991).

17. G. A. Koganov and R. Shuker, Phys. Rev. A 63, 015802 (2000).

18. A. Papoulis, Probability, Random Variables, and Stochastic Processes (McGraw-Hill, New York, 1991).
19. W. H. Press, S. A. Teukolsky, W. T. Vetterling, and B. P. Flannery, Numerical Recipes in C (Cambridge Univ. Press, Cambridge, 1992).

20. T. C. Ralph and C. M. Savage, Quantum Opt. 5, 113 (1993).

21. J. Arnaud, L. Chusseau, and F. Philippe, Phys. Rev. B 62, 13482 (2000).

22. J. Arnaud and M. Estéban, IEE Proc.-J: Optoelectron. 137, 55 (1990).

23. J. Arnaud, Electron. Lett. 29, 1320 (1993). 